\title{
The formation of composite film hydrogel membranes with polyamide layer
}

\author{
G.V. Yaculchak, N.M. Baran, Yu.Ya. Melnyk, Grytsenko O.M., Chopyk N.V.
}

Department of Plastic Engineering, Lviv Polytechnic National University, Ukraine, Lviv, 12, St. Bandera str., E-mail: natabmk@ukr.net

\begin{abstract}
The composite hydrogel membranes on the basis of 2-hydroxyethylmethacrylate (HEMA) and polyvinylpyrrolidone (PVP) copolymers modified by a thin layer of PA-6/PVP mixture by the diffusion deposition from the solution were synthesized. The influence of the surface adsorption of $P A-6 / P V P$ mixture on the physical and mechanical properties of the synthesized films has been investigated.

Keywords: membrane, hydrogel, 2-hydroxyethylmethacrylate, polyvinylpyrrolidone, polyamide-6, surface adsorption.
\end{abstract}

\section{Introduction}

Polymer hydrogels, which are close to biological cells in their physical state, have proven to be effective in medicine and pharmacy. However, in practice the wide usage of hydrogel products is limited by their low mechanical strength, even though generally the application of hydrogels has a great potential . Recently, the interest to the polymer hydrogel materials, that have high mechanical strength in a hydrated state along with the hydrophilic and diffusiontransport properties, has increased.

The forming of composite hydrogel materials as coatings or multilayer films is carried out in order to improve the strength of the films, reduce their thickness, obtain a defect-free surface layer, create films of complex configuration and special properties. The processing of composite films focuses on the ensuring the necessary interlayer adhesion during combin of polymer film materials.

\section{Results and Discussion}

The composite polymer membranes are obtained by their surface modification with the thin layer of another polymer. The method is based on applying the polymer solution on the surface of the semi-permeable membrane substrate. As a result of solution diffusion into the surface layer, deposition of the polymer modifier occurs.

The aliphatic polyamides based solutions are promising for polymer hydrogels modification. The obtained films are hydrophilic with improved mechanical strength.

Monomer-polymer compositions were dissolved in the aqueous medium to form hydrogel film membranes. The polimerization was performed with the potassium persulfate initiation. Obtained hydrogel films were rinsed in the distilled water for 24 hours to completely remove unreacted monomer and stored in the hydrated state.

The hydrogel films were modified by the thin layer of solution consisted of PA-6/PVP, formic acid and water. One-sided coating of the thin polyamide polymer layer on the hydrogel film surface was achieved as a result of its contact with the hydrogel sample surface for a defined period of time $-1 \div 10 \mathrm{~min}$, with further solvent evaporation under $75-80{ }^{\circ} \mathrm{C}$ for 30 minutes. Obtained composite hydrogel films were rinsed with water to complete removal of the solvent. In current study the films in the hydrated state were used.

\section{Conclusions}

The hydrogel membranes based on copolymers of 2-hydroxyethyl methacrylate and PVP, reinforced by the thin films as a result of PA-6/PVP polymer mixtures deposition from the solution, were synthesized. The dependence of their physical and mechanical properties on the surface adsorption was investigated. It was shown that the surface adsorption can be regulated by changing the hydrogel composition and the modifying polymer mixture as well as the holding time of hydrogel membrane in the modifying solution. 